Pacific Journal of Mathematics

SHARP ESTIMATES OF CONVOLUTION TRANSFORMS IN 


\title{
SHARP ESTIMATES OF CONVOLUTION TRANSFORMS IN TERMS OF DECREASING FUNCTIONS
}

\author{
GARY SAMPSON
}

Let $f * g$ denote the convolution transform of two Lebesgue measurable functions on the real line defined by the equation

$$
(f * g)(x)=\int_{-\infty}^{+\infty} f(t) g(x-t) d t .
$$

We get best possible upper and lower estimates for the expression

$$
\sup _{\substack{f_{i} \sim g_{i}^{*} \\|E| \leqq u}} \int_{E}\left|\left(f_{1} * \cdots * f_{n}\right)(x)\right|^{p} d(x)
$$

where $p=1$ and 2 , with applications to Fourier transform inequalities. Here $g_{i}^{*}$ are preassigned decreasing functions and the symbol $f_{i} \sim g_{i}^{*}$ means

$$
\left|\left\{x:\left|f_{i}(x)\right|>y\right\}\right|=\left|\left\{x: g_{i}^{*}(x)>y\right\}\right| \text { for all } y \text {. }
$$

o. Introduction. In order to formulate the general problem, we remember the Hardy and Littlewood estimate [4, page 130, Theorem 6.8$]$ of the $L_{q}$-norm $(q \geqq 2)$ for the Fourier coefficients $c_{n}=\int_{-\pi}^{\pi} f(t) e^{\text {int }}$ of $f$ in terms of the decreasing rearrangement $g^{*}$ of $|f|$ :

$$
\left\|c_{n}\right\|_{q} \leqq A_{q} \int_{0}^{2 \pi}\left(g^{*}(x)\right)^{q} x^{q-2} d(x)
$$

where the constant $A_{q}$ depends only on $q$. As one might expect, the same theorem [Theorem $E$, this paper] holds for the Fourier transform $\mathfrak{F}(f)$, i.e.

$$
\int_{-\infty}^{+\infty}|\mathfrak{S S}(f)|^{q} \leqq c(q) \int_{0}^{\infty}\left(g^{*}(x)\right)^{q} x^{q-2} d(x)
$$

holds for $q \geqq 2$, where $g^{*}$ is the decreasing rearrangement of $|f|$. It is very remarkable that for $(0.1)$, this is the best possible estimate in terms of $g^{*}$, i.e. independent of signs and arrangement of $f$, since for prescribed $g^{*}$ the left side always reaches the right side for some suitable $f$ and this same result is valid for $(0.2)$. Thus, Hardy and Littlewood were able to determine the

$$
\sup \|\mathfrak{F}(f)\|_{q}
$$

where $f$ varies over all functions with the same $g^{*}$. If $q=2 k$ (k 
positive integer), then we have, by using Parseval's formula, the following:

$$
\int|\mathfrak{F}(f)|^{2 k}=\sqrt{2 / \pi} \int|f * \cdots * f|^{2}
$$

Thus, we have the related problem of determining

$$
\sup \|f * \cdots * f\|_{2} \text {. }
$$

More generally, one should look for

$$
\sup \left\|T\left(f_{1}, f_{2}, \cdots, f_{k}\right)\right\|_{p},
$$

where $T$ denotes a multi-linear operator and the sup extends over all $f_{1}, \cdots, f_{k}$ with prescribed $g_{1}^{*}, \cdots, g_{k}^{*}$.

Recently, O'Neil obtained sharp bounds for $\int_{0}^{x} g^{*}$ in the case $g=$ $f_{1} * f_{2}$, which leads to sharp estimates for various norms of $g$. In the present paper, we obtain sharp bounds for $\int_{0}^{x} g^{*}$, where $g=f_{1} * \cdots * f_{k}$. We also obtain estimates for $\int_{0}^{x}\left(g^{*}\right)^{n}$ and $\int_{0}^{x}\left(g_{1} \cdots g_{n}\right)^{*}$ for the cases $n=2$ and $n=4$, where each of the $g$ 's is itself a convolution product. Because the estimates for the case $n=4$ are lengthy, we have omitted them from the present paper. Detailed estimates, all of which involve the combinations

$$
\frac{1}{x} \int_{0}^{\infty} f^{*}-f^{*}
$$

appear in Theorem 1.6, Theorem 2.1, and Corollary 3.3. In $\S 4$, we apply our results to give a new proof of some rearrangement theorems due to Hardy and Littlewood.

The functions $f, g, \cdots$ which appear in this paper will be Lebesgue measurable functions for which $|\{x:|f(x)|>y\}|<+\infty$ for every $y>0$. By the statement $f(x) \doteq g(x)$, we mean that $\mid\{x: f(x) \neq$ $g(x)\} \mid=0$. Theorems labeled with letters (e.g., Theorem A, Theorem $\mathrm{B}, \cdots$ ) are known; also, Theorem 2.2 (for the case $n=2$ ) and Corollary 1.8 are also known. So far as we know, all of the other Theorems and Corollaries are new.

1. Upper $L_{1}$-estimates. The estimates that we get will be in terms of the decreasing rearrangement $f^{*}$ and symmetrically decreasing rearrangement $\bar{f}$ of $|f(x)|$; where here, $f(x)$ is a complexvalued function and $x$ is a real parameter. Thus, we start by giving definitions of these ideas. 
Definition 1.1. The functions $f$ and $g$ are said to be equimeasurable, and we write $f \sim g$, if $|\{x:|f(x)|>y\}|=|\{x:|g(x)|>y\}|$ for all $y$.

Definition 1.2. By $f^{*}(x)$ we denote a function such that

(i) $f^{*} \sim|f|$.

(ii) $f^{*}(x)$ decreases for $x>0$.

Further, for $x>0$, we set

$$
f^{* *}(x)=\frac{1}{x} \int_{0}^{x} f^{*}(t) d t
$$

Definition 1.3. By $\bar{f}(x)$ we denote a function such that

(i ) $\bar{f} \sim|f|$

(ii) $\bar{f}(x)=\bar{f}(-x)$

(iii) $\bar{f}(x)$ decreases for $x>0$.

From the above definition, it follows that $f \sim g$, if and only if $\bar{f}(x) \doteq \bar{g}(x)$. Therefore, we get that $f^{*}(2|x|) \doteq \bar{f}(x)$, since $f^{*}(2|x|) \sim$ $\bar{f}(x)$ and $f^{*}(2|x|)$ is symmetrically decreasing.

The next lemma (Lemma 1.4) plays an important role in simplifying our proofs. We must first define the function,

$$
\varepsilon\left(x, \alpha, f^{*}\right)= \begin{cases}1 & x \in\left[0, \mid E_{\alpha} i\right] \\ 0 & \text { elsewhere }\end{cases}
$$

where $E_{\alpha}=\left\{t: f^{*}(t) \geqq f^{*}(\alpha)\right\}$.

I wish to thank W. B. Jurkat for suggesting the following lemma:

LEMMA 1.4 (Jurkat). If $\lim _{x \rightarrow+\infty} f^{*}(x)=0$ then

$$
f^{*}(x)=-\int_{0}^{\infty} \varepsilon\left(x, \alpha, f^{*}\right) d\left(f^{*}(\alpha)\right) .
$$

Proof. Given $x$, let $\alpha_{0}=\inf \left\{\alpha: f^{*}(\alpha)=f^{*}(x)\right\}$, then $f^{*}\left(\alpha_{0^{+}}\right)=$ $f^{*}(x)$. Hence,

$$
-\int_{0}^{\infty} \varepsilon\left(x, \alpha, f^{*}\right) d\left(f^{*}(\alpha)\right)=-\int_{\alpha_{0}+}^{\infty} d(f(\alpha))=f\left(\alpha_{0^{+}}\right)=f(x) .
$$

A nonnegative sequence $\left\langle\bar{a}_{n}\right\rangle_{n=-\infty}^{+\infty}$ is said to be symmetrically decreasing if $\bar{a}_{0} \geqq \bar{a}_{1}=\bar{a}_{-1} \geqq \cdots \geqq \bar{a}_{n}=\bar{a}_{-n} \geqq \cdots$. A well-known fact [1, Theorem 375, page 273] is that the convolution of symmetrically decreasing sequences is also a symmetrically decreasing sequence. The previous statement also holds if we replace the term "sequence" with the term "function". We see this in the next lemma. 
Lemma 1.5. The function $h_{n}=\bar{g}_{1} * \bar{g}_{2} * \cdots * \bar{g}_{n}$ is symmetrically decreasing.

Proof. To show that $h_{n}(x)=h_{n}(-x)$ is straight forward and we omit the proof. To show that $h_{n}$ decreases for $x>0$, we first consider the case where

$$
h_{2}(x)=\int_{-\infty}^{+\infty} \bar{g}_{1}(v) \bar{g}_{2}(x-v) d v
$$

and

$$
g_{1}^{*}(2 v)= \begin{cases}1 & 0 \leqq v \leqq u \\ 0 & \text { elsewhere }\end{cases}
$$

Then

$$
\begin{aligned}
h_{2}(x) & =\int_{0}^{\infty} g_{1}^{*}(2 v)\left\{\bar{g}_{2}(x-v)+\bar{g}_{2}(x+v)\right\} d v \\
& =\int_{0}^{u+x} d v g_{2}^{*}(2 v)+\int_{0}^{u-x} d v g_{2}^{*}(2 v) .
\end{aligned}
$$

For $x>0$, we see that $h_{2}(x)$ is decreasing.

Consider the case $\lim _{v \rightarrow \infty} g_{1}^{*}(2 v)=0$. We get after applying Lemma 1.4

$$
h_{2}(x)=-\int_{0}^{\infty} d\left(g_{1}^{*}(2 \alpha)\right) \int_{0}^{\infty} d v \varepsilon\left(v, \alpha, g_{1}^{*}\right)\left\{\bar{g}_{2}(x-v)+\bar{g}_{2}(x+v)\right\}
$$

and therefore, $h_{2}(x)$ decreases for $x>0$.

In the case where $\lim _{v \rightarrow \infty} g_{1}^{*}(2 v)=c$, we have

$$
h_{2}(x)=\int_{-\infty}^{+\infty}\left(\bar{g}_{1}(v)-c\right) \bar{g}_{2}(x-v) d v+c \int_{-\infty}^{+\infty} \bar{g}_{2}(v) d v .
$$

Therefore, it follows that $h_{2}(x)$ decreases for $x>0$ and hence $h_{n}(x)$ decreases for $x>0$.

Unless otherwise specified, the functions $f, g, \ldots$ which appear will be nonnegative. A remarkable inequality [1, page 279, Th. 379] can be formulated in the following manner:

THEOREM A. $\sup _{\substack{r_{1} \sim \bar{r} \\ f_{k} \sim \bar{g}_{k}}} \int_{-\infty}^{+\infty} d(x) r_{1}(x)\left(f_{1} * f_{2}\right)(x)=\int_{-\infty}^{+\infty} d(x) \bar{r}(x)\left(\bar{g}_{1} * \bar{g}_{2}\right)(x)$.

The following extension of Theorem A, which is well known for sequences, [1, page 273, Th. 374], will be of more interest to us. It can be stated in the following way: 
THEOREM B. $\sup _{\substack{r, \sim \\ f_{k} \sim \bar{g}}} \int_{-\infty}^{+\infty} d(x) r_{1}(x)\left(f_{1} * \cdots * f_{n}\right)(x)=\int_{-\infty}^{+\infty} d(x) \bar{r}(x) h_{n}(x)$.

Proof. At first, we see from Theorem A,

$$
\begin{aligned}
\sup _{\substack{r_{1} \sim \bar{r} \\
f_{k} \sim \bar{g}_{k}}} \int_{-\infty}^{+\infty} d(x) r_{1}(x)\left(f_{1} * f_{2}\right)(x) & =\sup _{f_{k} \sim \bar{g}_{k}} \int_{-\infty}^{+\infty} d(x) \bar{r}(x) \overline{\left(f_{1} * f_{2}\right)}(x) \\
& =\int_{-\infty}^{+\infty} d(x) \bar{r}(x)\left(\bar{g}_{1} * \bar{g}_{2}\right)(x) \\
& =\sup _{f_{k} \sim \bar{g}_{k}} \int_{-\infty}^{+\infty} d(x) \bar{r}(x)\left(f_{1} * f_{2}\right)(x) .
\end{aligned}
$$

Therefore, we see

$$
\begin{aligned}
\sup _{\substack{r, \sim \bar{T} \\
f_{k} \sim \bar{g}_{k}}} \int_{-\infty}^{+\infty} d(x) r_{1}(x)\left(f_{1} * f_{2} * f_{3}\right)(x) & =\sup _{f_{k} \bar{g}_{k}} \int_{-\infty}^{+\infty} d(x) \bar{r}(x)\left(\bar{g}_{1} * \overline{f_{2} * f_{3}}\right)(x) \\
& =\sup _{f_{k} \sim \bar{g}_{k}} \int_{-\infty}^{+\infty} d(x)\left(\bar{r} * \bar{g}_{1}\right)(x) \overline{\left(f_{2} * f_{3}\right)}(x) \\
& =\sup _{f_{k} \bar{g}_{k}} \int_{-\infty}^{+\infty} d(x)\left(\bar{r} * \bar{g}_{1}\right)(x)\left(f_{2} * f_{3}\right)(x) \\
& =\int_{-\infty}^{+\infty} d(x) \bar{r}(x)\left(\bar{g}_{1} * \bar{g}_{2} * \bar{g}_{3}\right)(x) .
\end{aligned}
$$

In general, we have

$$
\sup _{\substack{r_{1} \sim \overline{\bar{p}} \\ f_{k} \sim \bar{g}_{k}}} \int_{-\infty}^{+\infty} d(x) r_{1}(x)\left(f_{1} * \cdots * f_{n}\right)(x)=\int_{-\infty}^{+\infty} d(x) \bar{r}(x) h_{n}(x) .
$$

Our first new result is the following:

THEOREM 1.6. If $\lim _{x \rightarrow \infty} g_{k}^{*}(x)=\lim _{x \rightarrow \infty} r^{*}(x)=0$, and $r^{*}$, $g_{k}^{*}$ are finite a.e., for $1 \leqq k \leqq n$, then

$$
\sup _{\substack{r_{1} \sim r^{*} \\ f_{k} \sim g_{k}^{*}}} \int_{-\infty}^{+\infty} r_{1}(x)\left(f_{1} * \cdots * f_{n}\right)(x) \leqq \int_{0}^{\infty} d(x) x^{n-1}\left(r^{* *}-r^{*}\right)\left(g_{1}^{* *}-g_{1}^{*}\right) \cdots\left(g_{n}^{*}-g_{n}^{*}\right) .
$$

Proof. Case I. We will first show the theorem when,

$$
\begin{aligned}
& g_{k}^{*}(x)= \begin{cases}1 & 0 \leqq x \leqq a_{k} \\
0 & \text { elsewhere }\end{cases} \\
& r^{*}(x)= \begin{cases}1 & 0 \leqq x \leqq u \\
0 & \text { elsewhere }\end{cases}
\end{aligned}
$$

and where $0 \leqq a_{1} \leqq a_{2} \leqq \cdots \leqq a_{n}$. 
Suppose $u \leqq a_{n}$ :

$$
\begin{aligned}
& \int_{-\infty}^{+\infty} d(x) r_{1}(x) \int_{-\infty}^{+\infty} d t f_{n}(t)\left(f_{1} * \cdots * f_{n-1}\right)(x-t) \\
\leqq & \int_{-\infty}^{+\infty} d(x) r_{1}(x) \int_{-\infty}^{+\infty} d t\left(f_{1} * \cdots * f_{n-1}\right)(x-t) \\
= & \int_{-\infty}^{+\infty} d(x) r_{1}(x) \int_{-\infty}^{+\infty} d(x) f_{1}(x) \cdots \int_{-\infty}^{+\infty} d(x) f_{n-1}(x) \\
= & u a_{1} \cdots a_{n-1} \\
= & \int_{0}^{\infty} d(x) x^{n-1}\left(r^{* *}-r^{*}\right)\left(g_{1}^{* *}-g_{1}^{*}\right) \cdots\left(g_{n}^{* *}-g_{n}^{*}\right) .
\end{aligned}
$$

Suppose $u>a_{n}$ :

$$
\begin{aligned}
& \int_{-\infty}^{+\infty} d(x) r_{1}(x) \int_{-\infty}^{+\infty} d t f_{n}(t)\left(f_{1} * \cdots * f_{n-1}\right)(x-t) \\
\leqq & \int_{-\infty}^{+\infty} d(x) \int_{-\infty}^{+\infty} d t f_{n}(t)\left(f_{1} * \cdots * f_{n-1}\right)(x-t) \\
= & a_{1} \cdots a_{n} \\
= & \int_{0}^{\infty} d(x) x^{n-1}\left(r^{* *}-r^{*}\right)\left(g_{1}^{* *}-g_{1}^{*}\right) \cdots\left(g_{n}^{* *}-g_{n}^{*}\right) .
\end{aligned}
$$

Case II. First let us set,

$$
\begin{gathered}
\varepsilon_{0}(x)=\varepsilon(x, \alpha, \bar{r})+\varepsilon(-x, \alpha, \bar{r}) \\
\varepsilon_{1}(x)=\varepsilon\left(x, \beta, \bar{g}_{1}\right)+\varepsilon\left(-x, \beta, \bar{g}_{1}\right) \\
\vdots \\
\varepsilon_{n}(x)=\varepsilon\left(x, \gamma, \bar{g}_{n}\right)+\varepsilon\left(-x, \gamma, \bar{g}_{n}\right) \\
\varepsilon(x, \lambda, h)=\left\{\begin{array}{ll}
1 & 0 \leqq x \leqq\left|E_{\lambda}(h)\right| \quad \text { and } \quad E_{\lambda}(h)=\{t: h(t) \geqq h(\lambda)\} . \\
0 & \text { elsewhere }
\end{array} .\right.
\end{gathered}
$$

By Theorem B we have,

$$
\sup _{\substack{r_{1} \sim \bar{r} \\ f_{k} \sim \overline{g_{k}}}} \int_{-\infty}^{+\infty} d(x) r_{1}(x)\left(f_{1} * \cdots * f_{n}\right)(x)=\int_{-\infty}^{+\infty} d(x) \bar{r}(x)\left(\bar{g}_{1} * \cdots * \bar{g}_{n}\right)(x)
$$

by Lemma 1.4 we get,

$$
=(-1)^{n+1} \int_{0}^{\infty} d(\bar{r}(\alpha)) \int_{0}^{\infty} d\left(\bar{g}_{1}(\beta)\right) \cdots \int_{0}^{\infty} d\left(\bar{g}_{n}(\gamma)\right) \int_{-\infty}^{+\infty} d(x) \varepsilon_{0}(x)\left(\varepsilon_{1} * \cdots * \varepsilon_{n}\right)(x)
$$

and by Case I,

$$
\begin{aligned}
\leqq & (-1)^{n+1} \int_{0}^{\infty} d(\bar{r}(\alpha)) \int_{0}^{\infty} d\left(\bar{g}_{1}(\beta)\right) \cdots \int_{0}^{\infty} d\left(\bar{g}_{n}(\gamma)\right) \\
& \times \int_{0}^{\infty} d(x) x^{n-1}\left(\varepsilon_{0}^{* *}-\varepsilon_{0}^{*}\right) \cdots\left(\varepsilon_{n}^{* *}-\varepsilon_{n}^{*}\right) \\
= & \int_{0}^{\infty} d(x) x^{n-1}\left(r^{* *}-r^{*}\right)\left(g_{1}^{* *}-g_{1}^{*}\right) \cdots\left(g_{n}^{* *}-g_{n}^{*}\right) .
\end{aligned}
$$


If we combine Theorem B with Theorem 1.6, we find that

$$
\begin{aligned}
\int_{-\infty}^{+\infty} d(x) \bar{r}(x) h_{n}(x) & \leqq \int_{0}^{\infty} d(x) x^{n-1}\left(r^{* *}-r^{*}\right)\left(g_{1}^{* *}-g_{1}^{*}\right) \cdots\left(g_{n}^{* *}-g_{n}^{*}\right) \\
& =A\left(r^{*}, g_{1}^{*}, \cdots, g_{n}^{*}\right) .
\end{aligned}
$$

However, we do know more because of Theorem 2.1. Combining Theorem 1.6 with Theorem 2.1, we then have

$$
\int_{-\infty}^{+\infty} d(x) \bar{r}(x) h_{n}(x)=K \cdot A\left(r^{*}, g_{1}^{*}, \cdots, g_{n}^{*}\right),
$$

where $K$ is a constant $\left(1 / 2^{n} \leqq K \leqq 1\right)$ which depends on $r^{*}, g_{1}^{*}, \cdots, g_{n}^{*}$. In (\#), the right side (our estimate) has an advantage over the left side; that is, it is easier to determine. For example, take $g_{k}^{*}(t)=$ $1 / t^{\lambda_{k}}$ and $r^{*}(t)=1 / t^{\mu}$, where $0<\lambda_{k}, \mu<1$.

In applying our estimate (Theorem 1.6), the minus signs that appear could conceivably present difficulties; and moreover, the result only holds for functions whose decreasing rearrangement goes to 0 at infinity. The next estimate does away with these problems.

THEOREM 1.7.

$$
\begin{aligned}
& \sup _{\substack{|E| \leqq u \\
f_{k} \sim g_{k}^{*}}} \int_{-\infty}^{+\infty} d(x) \chi_{E}(x)\left(f_{1} * \cdots * f_{n}\right)(x) \\
\leqq & {\left[\int_{0}^{u} g_{1}^{*} \cdots \int_{0}^{u} g_{n}^{*}+\frac{u}{2} \sum_{m=1}^{n} \sum_{\substack{k=1 \\
k \neq m}}^{n} \int_{u}^{\infty} d(x) x^{n-2} g_{m}^{*} g_{k}^{*} \prod_{\substack{i=1 \\
i \neq m \\
i \neq k}} g_{i}^{* *}\right] }
\end{aligned}
$$

Proof. To prove the theorem, we will first show

$$
\begin{aligned}
& u \int_{u}^{\infty} d(x) x^{n-2}\left(g_{1}^{* *}-g_{1}^{*}\right) \cdots\left(g_{n}^{* *}-g_{n}^{*}\right) \\
\leqq & \int_{0}^{u} g_{1}^{*} \cdots \int_{0}^{u} g_{n}^{*}+\frac{u}{2} \sum_{m=1}^{n} \sum_{\substack{k=1 \\
k \neq m}}^{n} \int_{u}^{\infty} d(x) x^{n-2} g_{m}^{*} g_{k}^{*} \prod_{\substack{i=1 \\
i \neq m \\
i \neq k}}^{n} g_{i}^{* *}
\end{aligned}
$$

when $\lim _{x \rightarrow \infty} g_{k}^{*}(x)=0$, for each $1 \leqq k \leqq n$.

Case I. Here we show (1.1) when

$$
g_{k}^{*}(x)= \begin{cases}1 & 0 \leqq x \leqq a_{k} \\ 0 & \text { elsewhere }\end{cases}
$$

and $a_{1} \leqq a_{2} \leqq \cdots \leqq a_{n}$.

(A) $\left(u<a_{1}\right)$.

The left-side of (1.1) equals $u a_{1} \cdots a_{n-1}$.

The right-side of (1.1) equals 


$$
\begin{aligned}
\left\{u^{n}+\frac{u}{2} a_{1}^{n-1}+\cdots+\frac{u}{2}\left(a_{1} a_{2} \cdots a_{n-2}^{2}\right)-\frac{n}{2} u^{n}\right\} & +u a_{1} \cdots a_{n-1} \\
& >u a_{1} \cdots a_{n-1}
\end{aligned}
$$

(B) $\quad\left(a_{k} \leqq u \leqq a_{k+1}\right)$.

The left side of (1.1) equals $u a_{1} \cdots a_{n-1}$.

The right side of (1.1) equals

$$
\begin{aligned}
\left(a_{1} \cdots a_{k}\right)\left\{u^{n-k}+\frac{u}{2} a_{k+1}^{n-k-1}+\cdots+\frac{u}{2}\left(a_{k+1} \cdots a_{n-2}^{2}\right)\right. & \left.-\frac{(n-k)}{2} u^{n-k}\right\} \\
& +u a_{1} \cdots a_{n-1}
\end{aligned}
$$

$\geqq u a_{1} \cdots a_{n-1}$.

(C) $\quad\left(u>a_{n}\right)$

The left side of (1.1) equals $a_{1} \cdots a_{n}$.

The right side of $(1.1)$ equals $a_{1} \cdots a_{n}$.

Case II. Here we show (1.1) when $\lim _{x \rightarrow \infty} g_{k}^{*}(x)=0$, for each $1 \leqq k \leqq n$. First let me set,

$$
\begin{aligned}
\varepsilon_{1} & =\varepsilon\left(x, \alpha, g_{1}^{*}\right) \\
& \vdots \\
\varepsilon_{n} & =\varepsilon\left(x, \beta, g_{n}^{*}\right) .
\end{aligned}
$$

Applying Lemma 1.4 to the left side of (1.1), we find

$$
\begin{aligned}
& u \int_{u}^{\infty} d(x) x^{n-2}\left(g_{1}^{* *}-g_{1}^{*}\right) \cdots\left(g_{n}^{* *}-g_{n}^{*}\right) \\
= & (-1)^{n} u \int_{0}^{\infty} d\left(g_{1}^{*}(\alpha)\right) \cdots \int_{0}^{\infty} d\left(g_{1}^{*}(\beta)\right) \int_{u}^{\infty} d(x) x^{n-2}\left(\varepsilon_{1}^{* *}-\varepsilon_{1}^{*}\right) \cdots\left(\varepsilon_{n}^{* *}-\varepsilon_{n}^{*}\right)
\end{aligned}
$$

and by Case I,

$$
\begin{aligned}
\leqq & (-1)^{n} \int_{0}^{\infty} d\left(g_{1}^{*}(\alpha)\right) \cdots \int_{0}^{\infty} d\left(g_{n}^{*}(\beta)\right) \\
& \times\left[\int_{0}^{u} \varepsilon_{1}^{*} \cdots \int_{0}^{u} \varepsilon_{n}^{*}+\frac{u}{2} \sum_{m=1}^{n} \sum_{\substack{k=1 \\
k \neq m}}^{n} \int_{u}^{\infty} d(x) x^{n-2} \varepsilon_{m}^{*} \varepsilon_{k}^{*} \prod_{\substack{i=1 \\
i \neq m \\
i \neq k}}^{n} \varepsilon_{i}^{* *}\right] \\
= & \int_{0}^{u} g_{1}^{*} \cdots \int_{0}^{u} g_{n}^{*}+\frac{u}{2} \sum_{m=1}^{n} \sum_{\substack{k=1 \\
k \neq m}}^{n} \int_{u}^{\infty} d(x) x^{n-2} g_{m}^{*} g_{k}^{*} \sum_{\substack{i=1 \\
i \neq m \\
i \neq k}}^{n} g_{i}^{* *} .
\end{aligned}
$$

Therefore, by Theorem 1.6 with $r^{*}(x)=\left\{\begin{array}{ll}1 & 0 \leqq x \leqq u \\ 0 & \text { elsewhere }\end{array}\right.$ we have shown Theorem 1.7, when $\lim _{x \rightarrow \infty} g_{k}^{*}(x)=0$ for each $1 \leqq k \leqq n$.

Case III. Let us consider the case when $g_{1}^{*}$ is arbitrary and $\lim _{x \rightarrow \infty} g_{k}^{*}(x)=0$ for each $2 \leqq k \leqq n$. 
Define: $\quad \bar{g}_{1 r}(x)=\left\{\begin{array}{cl}\bar{g}_{1}(x) & |x| \leqq r \\ 0 & |x|>r\end{array}\right.$

Then, we find

$$
\begin{aligned}
& \int_{-u / 2}^{u / 2} d(v)\left(\bar{g}_{1} * \cdots * \bar{g}_{n}\right)(x) \\
\leqq & \lim _{r \rightarrow \infty} \int_{-\infty}^{+\infty} d t \bar{g}_{1 r}(t) \int_{-u / 2}^{u / 2} d(x)\left(\bar{g}_{2} * \cdots * \bar{g}_{n}\right)(x-t) \\
\leqq & \lim _{r \rightarrow \infty}\left[\int_{0}^{u} g_{1 r}^{*} \cdots \int_{0}^{u} g_{n}^{*}+\frac{u}{2} \sum_{m=2}^{n} \sum_{\substack{k=2 \\
k \neq m}}^{n} \int_{u}^{\infty} g_{m}^{*} g_{k}^{*} \int_{0}^{x} g_{1 r}^{*} \prod_{\substack{i=2 \\
i \neq n \\
i \neq k}}^{n} \int_{0}^{x} g_{i}^{*}\right. \\
& \left.+u \sum_{k=2}^{n} \int_{u}^{\infty} d(x) g_{1 r}^{*} g_{k}^{*} \prod_{\substack{i=2 \\
i \neq k}}^{n} \int_{0}^{x} g_{i}^{*}\right] \\
= & \int_{0}^{u} g_{1}^{*} \cdots \int_{0}^{u} g_{n}^{*}+\frac{u}{2} \sum_{j=1}^{n} \sum_{k=1}^{n} \int_{u}^{\infty} d(x) x^{n-2} g_{m}^{*} g_{k}^{*} \prod_{\substack{i=1 \\
i \neq m \\
i \neq k}}^{n} g_{i}^{* *} .
\end{aligned}
$$

Reapplying the above procedure and using induction, we find that the theorem holds when $\bar{g}_{1}, \cdots, \bar{g}_{n}$ are arbitrary.

A corollary of Theorem 1.7 is a result due to R. O'Neil [2, Lemma 1.5].

Corollary 1.8. (O'Neil's Lemma).

$$
\sup _{\substack{|E| \leq u \\ \text { f. } \\ g_{1} \sim g^{*}}} \int_{E}\left(f_{1} * g_{1}\right)(x) \leqq u^{2} f^{* *} g^{* *}+u \int_{u}^{\infty} f^{*} g^{*} .
$$

Proof. Simply take $n=2$ in Theorem 1.7 .

II. Lower $L_{1}$-estimates. In this section, we will show that the inequalities found in Theorem 1.6 and Theorem 1.7 are sharp. That is, the upper estimates become lower estimates when they are multiplied by suitable constants.

THEOREM 2.1. If $r^{* *}(x), g_{k}^{* *}(x)(1 \leqq k \leqq n)$ are finite for each $x$, then

$$
\begin{aligned}
& 2^{n-1} \sup _{\substack{r_{1} \sim r^{*} \\
f_{k} \sim g_{k}^{*}}} \int_{-\infty}^{+\infty} d(x) r_{1}(x)\left(f_{1} * \cdots * f_{n}\right)(x) \\
\geqq & \int_{0}^{\infty} d(x) x^{n-1}\left(r^{* *}-r^{*}\right)\left(g_{1}^{* *}-g_{1}^{*}\right) \cdots\left(g_{n}^{* *}-g_{n}^{*}\right) .
\end{aligned}
$$


Proof. We will first prove the theorem when,

$$
\begin{aligned}
& g_{k}^{*}(x)= \begin{cases}1 & 0 \leqq x \leqq a_{k} \\
0 & \text { elsewhere }\end{cases} \\
& r^{*}(x)= \begin{cases}1 & 0 \leqq x \leqq u \\
0 & \text { elsewhere }\end{cases}
\end{aligned}
$$

and where $a_{1} \leqq a_{2} \leqq \cdots \leqq a_{n}$.

Suppose $u \leqq a_{n}$ :

$$
\begin{aligned}
& \int_{-\infty}^{+\infty} d(x) \bar{r}(x) \int_{-\infty}^{+\infty} d t \bar{g}_{n}(t) h_{n-1}^{(x-t)} \\
= & \int_{0}^{u / 2} d(x)\left[2 \int_{0}^{a_{n} / 2-x} h_{n-1}+2 \int_{0}^{a_{n} / 2+x} h_{h-1}\right] \\
\geqq & \frac{u}{2} \int_{-a_{n} / 2}^{a_{n} / 2} d t h_{n-1} \\
\geqq & \frac{u}{2} \cdot \frac{a_{n-1}}{2} \int_{-a_{n} / 2}^{a_{n} / 2} d t h_{n-2} \\
\vdots & \frac{u \cdot a_{1} \cdots a_{n-1}}{2^{n-1}}=\frac{1}{2^{n-1}} \int_{0}^{\infty} d(x) x^{n-1}\left(r^{* *}-r^{*}\right)\left(g_{1}^{* *}-g_{1}^{*}\right) \cdots\left(g_{n}^{* *}-g_{n}^{*}\right) .
\end{aligned}
$$

When we are in the case $\left(u>a_{n}\right)$, we apply a similar procedure. Hence, we are finished when our functions are as in (2.1).

In the case where $\lim _{x \rightarrow \infty} g_{k}^{*}(x)=\lim _{x \rightarrow \infty} r^{*}(x)=0 \quad(1 \leqq k \leqq n)$, if we apply Lemma 1.4 as we did in Theorem 1.6 (Case II) then our result follows.

Now let us consider the case where $g_{1}^{*}$ and $r_{1}^{*}$ are arbitrary, and $\lim _{x \rightarrow \infty} g_{k}^{*}(x)=0$ for each $2 \leqq k \leqq n$.

Define:

$$
\begin{gathered}
g_{1 m}^{*}(x)=\left\{\begin{array}{cl}
g_{1}^{*}(x) & 0 \leqq x \leqq m \\
0 & x>m
\end{array}\right. \\
r_{s}^{*}(x)=\left\{\begin{array}{cl}
r^{*}(x) & 0 \leqq x \leqq s \\
0 & x>s
\end{array} .\right. \\
\int_{-\infty}^{+\infty} \bar{r}(x)\left(\bar{g}_{1} * \cdots * \bar{g}_{n}\right)(x) d(x) \\
=\lim _{m \rightarrow+\infty} \lim _{s \rightarrow+\infty} \int_{-\infty}^{+\infty} \bar{r}_{s}(x)\left(\bar{g}_{1 m}^{*} \cdots * \bar{g}_{n}\right)(x) d(x) \\
\geqq \frac{1}{2^{n-1}} \frac{\lim _{m \rightarrow+\infty}}{\lim _{s \rightarrow+\infty}} \int_{0}^{\infty} d(x) x^{n-1}\left(r_{s}^{* *}-r_{s}^{*}\right)\left(g_{1 m}^{* *}-g_{1 m}^{*}\right) \cdots\left(g_{n}^{* *}-g_{n}^{*}\right) \\
\geqq \frac{1}{2^{n-1}} \int_{0}^{\infty} d(x) x^{n-1}\left(r^{* *}-r^{*}\right)\left(g_{1}^{* *}-g_{1}^{*}\right) \cdots\left(g_{n}^{* *}-g_{n}^{*}\right) .
\end{gathered}
$$


If we apply the above procedure and use induction, then we are finished.

Our next theorem, Theorem 2.2, was known for the case $n=2$ by O'Neil [2].

THEOREM 2.2. If $g_{k}^{* *}(x)$ is finite for each $x(1 \leqq k \leqq n)$ and $n \geqq 2$, then

$$
\begin{aligned}
& n \cdot 2^{n-2} \sup _{\substack{|E| \leq u \\
f_{k} \sim g_{k}^{*}}} \int_{-\infty}^{+\infty} d(x) \chi_{E}(x)\left(f_{1} * \cdots * f_{n}\right)(x) \\
\geqq & \int_{0}^{u} g_{1}^{*} \cdots \int_{0}^{u} g_{n}^{*}+\frac{u}{2} \sum_{m=1}^{n} \sum_{\substack{k=1 \\
k \neq m}}^{n} \int_{u}^{\infty} d(x) x^{n-2} g_{k}^{*} g_{m}^{*} \prod_{\substack{i=1 \\
i \neq m \\
i \neq k}}^{n} g_{i}^{* *} .
\end{aligned}
$$

Proof. To prove the theorem, we will show

$$
\begin{aligned}
& \frac{n}{2} u \int_{u}^{\infty} d x x^{n-2}\left(g_{1}^{* *}-g_{1}^{*}\right) \cdots\left(g_{n}^{* *}-g_{n}^{*}\right) \\
\geqq & \int_{0}^{u} g_{1}^{*} \cdots \int_{0}^{u} g_{n}^{*}+\frac{u}{2} \sum_{m=1}^{n} \sum_{\substack{k=1 \\
k \neq m}}^{n} \int_{u}^{\infty} d(x) x^{n-2} g_{m}^{*} g_{k}^{*} \prod_{\substack{i=1 \\
i \neq m \\
i \neq k}}^{n} g_{i}^{* *}
\end{aligned}
$$

where

$$
g_{k}^{*}(x)= \begin{cases}1 & 0 \leqq x \leqq a_{k} \\ 0 & x>a_{k}\end{cases}
$$

and

$$
a_{1} \leqq a_{2} \leqq \cdots \leqq a_{n}
$$

(A) $\left(u<a_{1}\right)$

The left side of (2.2) equals $(n / 2) u a_{1} \cdots a_{n-1}$.

The right side of (2.2) equals

$$
\begin{aligned}
& \left(u^{n}+\frac{u}{2} a_{1}^{n-1}+\cdots+\frac{u}{2}\left(a_{1} \cdots a_{n-2}^{2}\right)-\frac{n}{2} u^{n}\right)+u a_{1} \cdots a_{n-1} \\
\leqq & \left(\frac{n-2}{2}\right)\left(u a_{1} \cdots a_{n-1}\right)+u a_{1} \cdots a_{n-1}=\frac{n}{2}\left(u a_{1} \cdots a_{n-1}\right) .
\end{aligned}
$$

(B) $\left(a_{k} \leqq u \leqq a_{k+1}\right)$.

The left side of (2.2) equals $(n / 2) u a_{1} \cdots a_{n-1}$.

The right side of (2.2) equals, 


$$
\begin{aligned}
\left(a_{1} \cdots a_{k}\right)\left\{u^{n-k}+\frac{u}{2} a_{k+1}^{n-k-1}+\cdots\right. & \left.+\frac{u}{2}\left(a_{k+1} \cdots a_{n-2}^{2}\right)-\frac{(n-k)}{2} u^{n-k}\right\} \\
& +u a_{1} \cdots a_{n-1} \leqq \frac{n}{2}\left(u a_{1} \cdots a_{n-1}\right) .
\end{aligned}
$$

(C) $\left(u>a_{n}\right)$.

The left side of $(2.2)$ equals $(n / 2) a_{1} \cdots a_{n}$.

The right side of (2.2) equals $a_{1} \cdots a_{n}$.

To complete the proof, we simply follow the same approach as we used in the proof of Theorem 2.1.

III. $L_{2}$-estimates. In this section, we are concerned with finding both upper and lower estimates of

$$
\sup _{\substack{f_{i} \sim g_{i}^{*} \\|E| \leqq u}} \int_{E}\left[\left(f_{1} * \cdots * f_{n}\right)(x)\right]^{p} d(x)
$$

where $p=2$. However, we were also able to get (explicitly) upper and lower estimates for the case $p=4$, but we have omitted them from this paper.

LEMMA 3.1.

$$
\begin{aligned}
& \sup _{\substack{|E| \leq u \\
f_{i j} \sim \bar{g}_{i j}}} \int_{-\infty}^{+\infty} d(x) \chi_{E}(x)\left(f_{11} * \cdots * f_{1 r}\right)(x)\left(f_{21} * \cdots * f_{2 s}\right)(x) \\
= & \int_{-u / 2}^{u / 2} d(x)\left(\bar{g}_{11} * \cdots * \bar{g}_{1 r}\right)(x)\left(\bar{g}_{21} * \cdots * \bar{g}_{2 s}\right)(x)
\end{aligned}
$$

Proof. We note that by Theorem B

$$
\begin{aligned}
& \int_{-\infty}^{+\infty} d(x) \chi_{E}(x)\left(f_{11} * \cdots * f_{1 r}\right)(x)\left(f_{21} * \cdots * f_{2 s}\right)(x) \\
\leqq & \int_{-\infty}^{+\infty} d(x) \overline{\chi_{E}\left(f_{11} * \cdots * f_{1 r}\right)}(x)\left(\bar{g}_{21} * \cdots * \bar{g}_{2 s}\right)(x) \\
\leqq & \int_{-\infty}^{+\infty} d(x) \bar{\chi}_{E}(x) \overline{\left(f_{11} * \cdots * f_{1 r}\right)}(x)\left(\bar{g}_{21} * \cdots * \bar{g}_{2 s}\right)(x) \\
\leqq & \int_{-\infty}^{+\infty} d(x) \bar{\chi}_{E}(x)\left(\bar{g}_{11} * \cdots * \bar{g}_{1 r}\right)(x)\left(\bar{g}_{21} * \cdots * \bar{g}_{2 s}\right)(x) .
\end{aligned}
$$

We set,

$$
\Delta_{1}(x)=x^{r}\left(g_{11}^{* *}-g_{11}^{*}\right) \cdots\left(g_{1 r}^{* *}-g_{1 r}^{*}\right)
$$

and

$$
\Delta_{2}(x)=x^{8}\left(g_{21}^{* *}-g_{21}^{*}\right) \cdots\left(g_{2 s}^{* *}-g_{2 s}^{*}\right)
$$


THEOREM 3.2. If $g_{i j}^{* *}(x)$ are finite for each $x$ and $\lim _{x \rightarrow \infty} g_{i j}^{*}(x)=0$, then there exists a constant $K, 2^{-r-s} \leqq K \leqq 1$, such that

$$
\begin{aligned}
& \sup _{\substack{|E| \leq u \\
\text { fij } \\
f_{i j} g_{i j}^{*}}} \int_{-\infty}^{+\infty} d(x) \chi_{E}(x)\left(f_{11} * \cdots * f_{1 r}\right)(x)\left(f_{21} * \cdots * f_{2 s}\right)(x) \\
= & K \cdot\left[\int_{0}^{u} \frac{d(x)}{x^{2}}\left(\Delta_{1}(x) \cdot \Delta_{2}(x)\right)+u\left(\int_{u}^{\infty} \frac{d(x)}{x^{2}} \Delta_{1}(x)\right)\left(\int_{u}^{\infty} \frac{d(x)}{x^{2}} \Delta_{2}(x)\right] .\right.
\end{aligned}
$$

Here, $K$ depends on $g_{i j}^{*}$ and $u$; and we note that $1 \leqq i \leqq 2$ and $1 \leqq j \leqq \max (r, s)$.

Proof. (i) We first prove that $K \leqq 1$. We consider the case where,

$$
\begin{gathered}
g_{i j}^{*}(x)= \begin{cases}1 & 0 \leqq x \leqq a_{i j} \\
0 & \text { elsewhere }\end{cases} \\
a_{11} \leqq \cdots \leqq a_{1 r} \text { and } a_{21} \leqq \cdots \leqq a_{2 s} .
\end{gathered}
$$

Without loss of generality, we can assume that $a_{1 r} \leqq a_{2 s}$ and $a_{1 r}>0$. (A) $\left(u \geqq a_{2 s}\right)$

$$
\begin{aligned}
& \int_{-u / 2}^{u / 2} d(x)\left(\bar{g}_{11} * \cdots * \bar{g}_{1 r}\right)(x)\left(\bar{g}_{21} * \cdots * \bar{g}_{2 s}\right)(x) \\
\leqq & \frac{a_{21} \cdots a_{2 s}}{a_{2 s}} \int_{-\infty}^{+\infty} d(x)\left(\bar{g}_{11} * \cdots * \bar{g}_{1 r}\right)(x) \\
= & \frac{a_{11} \cdots a_{1 r} a_{21} \cdots a_{2 s}}{a_{2 s}} \\
= & \int_{0}^{u} \frac{d(x)}{x^{2}}\left(\Delta_{1}(x) \Delta_{2}(x)\right)+u\left(\int_{u}^{\infty} \frac{d(x)}{x^{2}} \Delta_{1}(x)\right)\left(\int_{u}^{\infty} \frac{d(x)}{x^{2}} \Delta_{2}(x)\right)
\end{aligned}
$$

(B) $\quad\left(a_{1 r} \leqq u<a_{2 s}\right)$

$$
\begin{aligned}
& \int_{-u / 2}^{u / 2} d(x)\left(\bar{g}_{11} * \cdots * \bar{g}_{1 r}\right)(x)\left(\bar{g}_{21} * \cdots * \bar{g}_{2 s}\right)(x) \\
\leqq & \frac{a_{11} \cdots a_{1 r} a_{21} \cdots a_{2 s}}{a_{2 s}} \\
= & u\left(\int_{u}^{\infty} \frac{d(x)}{x^{2}} \Delta_{1}(x)\right)\left(\int_{u}^{\infty} \frac{d(x)}{x^{2}} \Delta_{2}(x)\right) .
\end{aligned}
$$

(C) $\left(u<a_{1 r}\right)$

$$
\begin{aligned}
& \int_{-u / 2}^{u / 2} d(x)\left(\bar{g}_{11} * \cdots * \bar{g}_{1 r}\right)(x)\left(\bar{g}_{21} * \cdots * \bar{g}_{2 s}\right)(x) \\
\leqq & \frac{u\left(a_{11} \cdots a_{1 r}\right)\left(a_{21} \cdots a_{2 s}\right)}{a_{1 s} \cdot a_{2 s}}=u\left(\int_{u}^{\infty} \frac{d(x)}{x^{2}} \Delta_{1}(x)\right)\left(\int_{u}^{\infty} \frac{d(x)}{x^{2}} \Delta_{2}(x)\right) .
\end{aligned}
$$


To complete the proof, we apply Lemma 1.4 as we did in Theorem 1.6 (Case II).

(ii) Here we prove that $K \geqq 2^{-r-s}$. First, we consider functions as defined by (3.1).

$$
\begin{aligned}
\text { (A) } \quad & \left(u \geqq a_{2 s}\right) \\
& \int_{-u / 2}^{u / 2} d(x)\left(\bar{g}_{11} * \cdots * \bar{g}_{1 r}\right)(x)\left(\bar{g}_{21} * \cdots * \bar{g}_{2 s}\right)(x) \\
= & 2 \int_{0}^{a_{1 r} / 2} d(x)\left[\int_{0}^{(u / 2)-x} h_{1(r-1)}+\int_{0}^{(u / 2)+x} h_{1(r-1)}\right]\left[\int_{0}^{\left(a_{2 s} / 2\right)-x} h_{2(s-1)}+\int_{0}^{\left(a_{2 s / 2}\right) \div x} h_{2(s-1)}\right] \\
\geqq & a_{1 r} \int_{0}^{u / 2} h_{1(r-1)} \int_{0}^{a_{2 s} / 2} h_{2(s-1)} \geqq \frac{a_{11} \cdots a_{1 r} a_{21} \cdots a_{2 s}}{2^{r+s} a_{2 s}} \\
= & \frac{1}{2^{(r+s)}}\left[\int_{0}^{u} \frac{d(x)}{x^{2}}\left(\Delta_{1}(x) \cdot \Delta_{2}(x)\right)+u\left(\int_{u}^{\infty} \frac{d(x)}{x^{2}} \Delta_{1}(x)\right)\left(\int_{u}^{\infty} \frac{d(x)}{x^{2}} \Delta_{2}(x)\right)\right] .
\end{aligned}
$$

(B) $\quad\left(a_{1 r} \leqq u<a_{2 s}\right)$

$$
\begin{aligned}
& \int_{-u / 2}^{u / 2} d(x)\left(\bar{g}_{11} * \cdots * \bar{g}_{1 r}\right)(x)\left(\bar{g}_{21} * \cdots * \bar{g}_{2 s}\right)(x) \\
= & \int_{0}^{a_{1 r / 2}} d(x)\left[\int_{0}^{(u / 2)-x} h_{1(r-1)}+\int_{0}^{(u / 2)+x} h_{1(r-1)}\right]\left[\int_{0}^{\left(a_{2 s} / 2\right)-x} h_{2(s-1)}+\int_{0}^{\left(a_{2 s} / 2\right) \div x} h_{2(s-1)}\right] \\
\geqq & \frac{a_{11} \cdots a_{1 r} a_{21} \cdots a_{2 s}}{2^{(r+s)} a_{2 s}} \\
= & \left(\frac{1}{2^{r+s}}\right)\left(u\left(\int_{u}^{\infty} \frac{d(x)}{x^{2}} \Delta_{1}(x)\right)\left(\int_{u}^{\infty} \frac{d(x)}{x^{2}} \Delta_{2}(x)\right)\right) .
\end{aligned}
$$

(C) $\quad\left(u<a_{1 r}\right)$

$$
\begin{aligned}
& \int_{-u / 2}^{u / 2} d(x)\left(\bar{g}_{11} * \cdots * \bar{g}_{1 r}\right)(x)\left(\bar{g}_{21} * \cdots * \bar{g}_{2 s}\right)(x) \\
= & 2 \int_{0}^{u / 2} d(x)\left[\int_{0}^{\left(a_{1 r} / 2-x\right.} h_{1(r-1)}+\int_{0}^{\left(a_{1 r} / 2\right)+x} h_{1(r-1)}\right]\left[\int_{0}^{\left(a_{2 s} / 2\right)-x} h_{2(s-1)}+\int_{0}^{\left(a_{2 s} / 2\right)+x} h_{2(s-1)}\right] \\
\geqq & \frac{u a_{11} \cdots a_{1 r} a_{21} \cdots a_{2 s}}{2^{(r+s)} a_{1 r} a_{2 s}} \\
= & \left(\frac{1}{2^{r+s}}\right)\left(u\left(\int_{u}^{\infty} \frac{d(x)}{x^{2}} \Delta_{1}(x)\right)\left(\int_{u}^{\infty} \frac{d(x)}{x^{2}} \Delta_{2}(x)\right) .\right.
\end{aligned}
$$

To complete the proof, we apply Lemma 1.4 as we did in Theorem 1.6 (Case II).

COROLLARY 3.3. If $g_{k}^{* *}(x)$ are finite for each $x$ and $\lim _{x \rightarrow \infty} g_{k}^{*}(x)=$ $0,1 \leqq k \leqq n$, then there exists a constant $K, 2^{-2 n} \leqq K \leqq 1$, such that 


$$
\begin{aligned}
& \sup _{\substack{|E| \leq u \\
f_{j} \sim g_{j}^{*}}} \int_{-\infty}^{+\infty} d(x) \chi_{E}(x)\left[\left(f_{1} * \cdots * f_{n}\right)(x)\right]^{2} \\
= & K\left[\int_{0}^{u} d(x) x^{2(n-1)}\left(g_{1}^{* *}-g_{1}^{*}\right)^{2} \cdots\left(g_{n}^{* *}-g_{n}^{*}\right)^{2}\right. \\
& \left.+u\left(\int_{u}^{\infty} d(x) x^{n-2}\left(g_{1}^{* *}-g_{1}^{*}\right) \cdots\left(g_{n}^{* *}-g_{n}^{*}\right)\right)^{2}\right] .
\end{aligned}
$$

Here $K$ depends on $g_{k}^{*}(1 \leqq k \leqq n)$ and $u$.

Proof. This is a special case of Theorem 3.2, with $r=s=n$ and $g_{i i}^{*}=g_{i}^{*}$.

IV. Applications. The functions $f(x)$ in this section are complexvalued and the variable $x$ is real. We require that $\operatorname{Re}(f(x))$ and $\operatorname{Im}(f(x))$ be Lebesgue measurable and $|\{x:|f(x)|>y\}|<\infty$ for every $y>0$. In a natural way we can now talk about the decreasing rearrangement function $f^{*}$ of $f$.

We mean by the statement

$$
f(x)={ }_{\left(L_{p}\right)} \lim _{n \rightarrow \infty} f_{n}(x), x \in(-\infty, \infty),
$$

that $\lim _{n \rightarrow \infty}\left\|f_{n}-f\right\|_{p}=0$.

By Plancherel's theorem if we let $f \in L_{2}(-\infty,+\infty)$, then $\mathfrak{F}(f)(x)={ }_{\left(L_{2}\right)} 1 / \sqrt{2 \pi} \lim _{n \rightarrow \infty} \int_{-n}^{n} f(t) e^{i t x} d t$, exists a.e., and belongs to $L_{2}$; also, if $f \in L_{2}$, then $\|\mathfrak{F}(f)\|_{2}=\|f\|_{2}$. A generalization of Plancherel's theorem (Theorem C and D), due to Hardy and Littlewood, is a consequence of Corollary 3.3. Another rearrangement theorem [4, pages 128-131], due to Hardy and Littlewood, is contained in Theorem E.

THEorem C. (Hardy and Littlewood). If $|f(x)|^{q}|x|^{q-2} \quad(q \geqq 2)$ belongs to $L(-\infty,+\infty)$, then $\mathfrak{F}(f)(x)={ }_{\left(L_{q}\right)} 1 / \sqrt{2 \pi} \lim _{n \rightarrow \infty} \int_{-n}^{n} f(t) e^{i t x} d t$, exists a.e., and belongs to $L_{q}$; and

$$
\|\mathfrak{F}(f)\|_{q} \leqq q\left\{\int_{-\infty}^{+\infty}|f(x)|^{q}|x|^{q-2} d(x)\right\}^{1 / q}
$$

Proof. Suppose first that $f \in L_{\infty}$ and has compact support, then we have by Plancherel's theorem,

$$
\begin{aligned}
\int_{-\infty}^{+\infty}|\mathfrak{f}(f)|^{2 n} d(x) & =\int_{-\infty}^{+\infty}|f * f * \cdots * f|^{2} d(x) \\
& \leqq \int_{-\infty}^{+\infty}(|f| *|f| * \cdots *|f|)^{2} d(x)
\end{aligned}
$$


and by Corollary 3.3,

$$
\begin{aligned}
& \leqq \int_{0}^{\infty} d(x) x^{2(n-1)}\left(f^{* *}-f^{*}\right)^{2 n} \\
& \leqq(2 n)^{2 n} \int_{0}^{\infty} d(x) x^{2(n-1)}\left(f^{*}(x)\right)^{2 n} \\
& \leqq(2 n)^{2 n} \int_{-\infty}^{+\infty} d(x) x^{2(n-1)}|f(x)|^{2 n}
\end{aligned}
$$

We first note that $M_{q, \nu}=\left\{x g(x): \int_{-\infty}^{+\infty} d(\nu(x))|x g(x)|^{q}<+\infty\right.$, where $\left.d(\nu(x))=d(x) / x^{2}\right\}=L_{q, \nu}(-\infty,+\infty)$. Now for each $h \in M_{q, \nu}$ set $T(h)=$ $\mathfrak{\mho}(h / x)$. Here, we can apply the interpolation theorem of RieszThorin to the operator $T$ [4, pages 93-96].

Theorem D. (Hardy and Littlewood). If $f \in L_{p}(1<p \leqq 2)$, then

$$
\left\{\int_{-\infty}^{+\infty}|\mathfrak{F}(f)(x)|^{p}|x|^{p-2} d(x)\right\}^{1 / p} \leqq q\|f\|_{p}
$$

where $1 / p+1 / q=1$.

Proof. Use our estimate from Theorem C for $\|\mathfrak{F}(f)\|_{q}$, then use the proof found in [3, Theorem 80, page 110].

If you take $f(t)=1 /(|t|+1), t \in(-\infty,+\infty)$, then $\|\mathfrak{F}(f)\|_{\infty}=\infty$ and $\|t f(t)\|_{\infty}=1$. Thus, Theorem $\mathrm{C}$ does not hold when $q=\infty$. However we see from Corollary 3.3 that if $f \in L_{1} \cap L_{\infty}$, then

$$
\|\mathfrak{F}(f)\|_{\infty} \leqq\left\|x\left(f^{* *}-f^{*}\right)\right\|_{\infty} \text {. }
$$

We define the

$$
\text { set } A_{q}=\left\{g^{*}: \int_{0}^{\infty}\left(g^{*}(x)\right)^{q} x^{q-2} d(x)<+\infty \text {, and } \lim _{x \rightarrow \infty} g^{*}(x)=0\right\} \text {. }
$$

THEOREM E. (Hardy and Littlewood). $g^{*} \in A_{q}$ if and only if $\mathfrak{F}(f) \in L_{q}(q \geqq 2)$, for every $f \sim g^{*}$. If $g^{*} \in A_{q}(q \geqq 2)$, then

$$
\|\mathfrak{F}(f)\|_{q} \leqq c(q)\left\{\int_{0}^{\infty}\left(g^{*}(x)\right)^{q} x^{q-2} d(x)\right\}^{1 / q}
$$

for every $f \sim g^{*}$ where $c(q)$ is a constant which depends on $q$.

Proof. $\quad(\Longleftarrow:)$

Lemma. If $\mathfrak{F}(\bar{f}) \in L_{q}(q \leqq 2)$, then 


$$
\left\{\int_{-\infty}^{+\infty} d(x)|x|^{q-2}|\bar{f}(x)|^{q}\right\}^{1 / q} \leqq c(q)\|\mathfrak{F}(\bar{f})\|_{q} .
$$

Proof. Suppose $f \in L_{1} \cap L_{\infty}$, then by Plancherel's theorem

$$
\begin{aligned}
\int_{-\infty}^{+\infty}|\mathfrak{\mho}(\bar{f})|^{2 n} & =\int_{-\infty}^{+\infty}(\bar{f} * \cdots * \bar{f})^{2} d(x) \\
& \geqq \int_{-\infty}^{+\infty} x^{2(n-1)}|\bar{f}(x)|^{2 n} d(x) .
\end{aligned}
$$

To complete the proof, we note [4, page 19] that if $\sum_{0}^{\infty} a_{n} \cos (n x) \in L_{q}$ $(q \geqq 2)$ for $a_{1} \geqq a_{2} \geqq \cdots \geqq a_{n} \geqq \cdots$, then

$$
\int_{0}^{\pi}\left|\sum_{0}^{\infty} a_{n} \cos (n x)\right|^{q} d(x) \geqq B(q) \sum_{v}^{\infty} a_{n}^{q} n^{q-2} \text {. }
$$

We quote from Titchmarsh [3, pages 70, 71, 109]. Let

$$
a_{\nu}=\int_{\nu / \lambda}^{(\nu+1) / \lambda} \bar{f}(t) d t \quad(\nu=0, \pm 1, \pm 2, \cdots)
$$

and

$$
\Phi_{n}(x)=\sum_{\nu=-n}^{n} a_{\nu} e^{i \nu x / \lambda}
$$

Then, if $b>0$ and $n=[\lambda b]-1$,

$$
\lim _{\lambda \rightarrow \infty} \Phi_{n}(x)=\int_{-b}^{b} \bar{f}(t) e^{i x t} d t
$$

uniformly in any finite interval. We note that in our case $a_{0}=$ $a_{-1} \geqq a_{1}=a_{-2} \geqq \cdots \geqq a_{n} \geqq 0$. From (4.1) we see that,

$$
B(q) \int_{-\pi \lambda}^{\pi \lambda}\left|\Phi_{n}(x)\right|^{q} d(x)=\lambda \int_{-\pi}^{\pi}\left|\sum_{\nu=-n}^{n} a_{\nu} e^{i \nu x}\right|^{q} d(x) \geqq \lambda \sum_{\nu=-n}^{n} a_{\nu}^{q}|\nu|^{q-2} .
$$

If $\nu \geqq 1$, then

$$
a_{\nu}^{q} \geqq[\bar{f}(\nu+1 / \lambda)]^{q} \cdot \frac{1}{\lambda^{q}} \geqq \frac{1}{2 \lambda(\nu+1)^{q-2}} \int_{(\nu+1) / \lambda}^{(\nu+2) / \lambda}(\bar{f}(t))^{q} t^{q-2} d t .
$$

Since,

$$
\left\|\int_{-b}^{b} \bar{f}(t) e^{i t x} d t-\int_{-a}^{a} \bar{f}(t) e^{i t x} d t\right\|_{q} \leqq \int_{a<|x|<b}|\bar{f}(x)|^{q}|x|^{q-2} d(x)
$$

we get that

$$
\lim _{b \rightarrow \infty}\left\|\mathfrak{F}(\bar{f})-\int_{-b}^{b} \bar{f}(t) e^{i t x} d t\right\|_{q}=0
$$


Hence, we get out result.

$\Leftrightarrow$ : In the proof of Theorem $C$ we showed that if $f \in L_{\infty}$ and has compact support, then

$$
\int_{-\infty}^{+\infty}|\mathfrak{F}(f)|^{2 n} d(x) \leqq(2 n)^{2 n} \int_{0}^{\infty} d(x) x^{2(n-1)}\left(g^{*}(x)\right)^{2 n} .
$$

Now if $f$ is arbitrary $\left(f \sim g^{*}\right)$, then we define

$$
f_{r}(x)=\left\{\begin{array}{cl}
f(x) & |f(x)| \leqq r,|x| \leqq r \\
r & |f(x)|>r,|x| \leqq r \\
0 & \text { elsewhere }
\end{array}\right.
$$

therefore $\left(\left|f-f_{r}\right|\right)^{*}(x) \leqq g^{*}(x)$ and $\lim _{r \rightarrow \infty}\left(\left|f-f_{r}\right|\right)^{*}(x)=0$. Hence it follows that,

$$
\int_{-\infty}^{+\infty}|\mathfrak{F}(f)|^{2 n} d(x) \leqq(2 n)^{2 n} \int_{0}^{\infty} d(x) x^{2(n-1)}\left(g^{*}(x)\right)^{2 n}
$$

Next, we show the theorem for $2<q<2 n$.

Let

$$
h_{1}(x)= \begin{cases}x f(x) & |x f(x)| \leqq 3 \lambda \\ \lambda e^{i \arg (x f(x))} & |x f(x)|>3 \lambda\end{cases}
$$

and

$$
\begin{gathered}
h_{2}(x)=\left\{\begin{array}{cc}
0 & |x f(x)| \leqq 3 \lambda \\
x f(x)-\lambda e^{i \mu r g(x f(x))} & |x f(x)|>3 \lambda .
\end{array}\right. \\
\|\mathfrak{F}(h / x)\|_{q}^{q}=q \cdot 2^{q} \int_{0}^{\infty} \lambda^{q-1}|\{x:|\mathfrak{F}(h / x)|>2 \lambda\}| d(\lambda) \\
\leqq c(n, q ; 2)\left[\int_{0}^{\infty} \lambda^{q-2 n-1} \int_{0}^{\infty} y^{2 n-1} \nu\left\{x: x f_{1}^{*}(x)>y\right\}+\int_{0}^{\infty} \lambda^{q-3} \int_{0}^{\infty} y \nu\left\{x: x f_{2}^{*}(x)>y\right\}\right] \\
\leqq c(n, q ; 2)\left[\int_{0}^{\infty} \lambda^{q-2 n-1} \int_{0}^{3 \lambda} y^{2 n-1} \nu\left\{x: x g^{*}(x)>y\right\}+\int_{0}^{\infty} \lambda^{q-3} \int_{0}^{\infty} y \nu\left\{x: x f_{2}^{*}(x)>y\right\}\right] \\
\leqq c(n, q ; 2)\left[\int_{0}^{\infty} \lambda^{q-1} \nu\left\{x: x g^{*}(x)>\lambda\right\}\right] \leqq c(n, q, 2)\left\|x g^{*}(x)\right\|_{q, 2}^{q} .
\end{gathered}
$$

Acknowledgement. I would like to thank W. B. Jurkat for bringing this problem to my attention and whose help and encouragement made this paper possible.

I would also like to thank Edwin Hewitt for his helpful comments.

\section{REFERENCES}

1. Hardy, Littlewood, and Polya, Inequalities, Cambridge at the Univ. Press. London, 1934. 
2. R. O'Neil, Conv. Operators and $L(p, q)$ spaces, Duke Math. J., 30 (1963).

3. E. C. Titchmarsh, Intro. to the Theory of Fourier Integrals, Oxford Univ. Press, 2nd edition 1962.

4. A. Zygmund, Trig. Series, Vol. II, Cambridge at the Univ. Press, London, 1959.

Received November 18, 1970.

SYRACUSE UNIVERSITY

AND

California Institute of Technology 



\section{PACIFIC JOURNAL OF MATHEMATICS}

\section{EDITORS}

H. SAMELSON

Stanford University

Stanford, California 94305

C. R. Hовву

University of Washington

Seattle, Washington 98105
J. DUGUNDJI

Department of Mathematics

University of Southern California

Los Angeles, California 90007

RICHARD ARENS

University of California

Los Angeles, California 90024

\section{ASSOCIATE EDITORS}
E. F. BECKENBACH
B. H. NeumanN
F. WOLF
K. YoSHIDA

\section{SUPPORTING INSTITUTIONS}

UNIVERSITY OF BRITISH COLUMBIA

CALIFORNIA INSTITUTE OF TECHNOLOGY

UNIVERSITY OF CALIFORNIA

MONTANA STATE UNIVERSITY

UNIVERSITY OF NEVADA

NEW MEXICO STATE UNIVERSITY

OREGON STATE UNIVERSITY

UNIVERSITY OF OREGON

OSAKA UNIVERSITY

UNIVERSITY OF SOUTHERN CALIFORNIA
STANFORD UNIVERSITY

UNIVERSITY OF TOKYO

UNIVERSITY OF UTAH

WASHINGTON STATE UNIVERSITY

UNIVERSITY OF WASHINGTON

AMERICAN MATHEMATICAL SOCIETY CHEVRON RESEARCH CORPORATION NAVAL WEAPONS CENTER 


\section{Pacific Journal of Mathematics}

\section{Vol. 38, No. $1 \quad$ March, 1971}

Bruce Alan Barnes, Banach algebras which are ideals in a Banach algebra ..... 1

David W. Boyd, Inequalities for positive integral operators............... 9

Lawrence Gerald Brown, Note on the open mapping theorem .............. 25

Stephen Daniel Comer, Representations by algebras of sections over Boolean

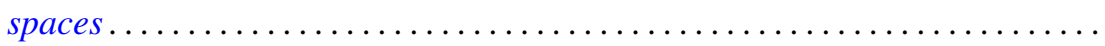

John R. Edwards and Stanley G. Wayment, On the nonequivalence of

conservative Hausdorff methods and Hausdorff moment sequences ........

P. D. T. A. Elliott, On the limiting distribution of additive functions $(\bmod 1) \ldots \ldots$

Mary Rodriguez Embry, Classifying special operators by means of subsets

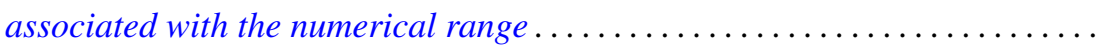

Darald Joe Hartfiel, Counterexamples to a conjecture of G. N. de Oliveira ......

C. Ward Henson, A family of countable homogeneous graphs...............

Satoru Igari and Shigehiko Kuratsubo, A sufficient condition for

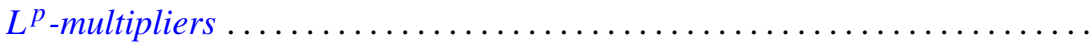

William A. Kirk, Fixed point theorems for nonlinear nonexpansive and

generalized contraction mappings............................

Erwin Kleinfeld, A generalization of commutative and associative rings ...... 95

D. B. Lahiri, Some restricted partition functions. Congruences modulo $11 \ldots \ldots 103$

T. Y. Lin, Homological algebra of stable homotopy ring $\pi *$ of spheres ....... 117

Morris Marden, A representation for the logarithmic derivative of a meromorphic function...........................

John Charles Nichols and James C. Smith, Examples concerning sum properties for metric-dependent dimension functions . .

Asit Baran Raha, On completely Hausdorff-completion of a completely

Hausdorff space.

M. Rajagopalan and Bertram Manuel Schreiber, Ergodic automorphisms and affine transformations of locally compact groups..........

N. V. Rao and Ashoke Kumar Roy, Linear isometries of some function

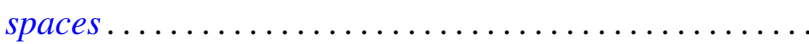

William Francis Reynolds, Blocks and F-class algebras of finite groups

Richard Rochberg, Which linear maps of the disk algebra are multiplicative ...

Gary Sampson, Sharp estimates of convolution transforms in terms of decreasing

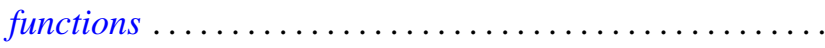

Stephen Scheinberg, Fatou's lemma in normed linear spaces

Ken Shaw, Whittaker constants for entire functions of several complex

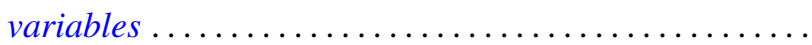

James DeWitt Stein, Two uniform boundedness theorems................ 251

$\mathrm{Li} \mathrm{Pi} \mathrm{Su,} \mathrm{Homomorphisms} \mathrm{of} \mathrm{near-rings} \mathrm{of} \mathrm{continuous} \mathrm{functions} \mathrm{.} \mathrm{.............} 261$

Stephen Willard, Functionally compact spaces, $C$-compact spaces and mappings of minimal Hausdorff spaces....................... 